\title{
On the scaling of fragmentation and energy dissipation in collisions of dust aggregates
}

\author{
Philipp Umstätter ${ }^{1} \cdot$ Herbert M. Urbassek ${ }^{1}$ (1)
}

Received: 17 August 2020 / Accepted: 10 February 2021 / Published online: 21 March 2021

(c) The Author(s) 2021

\begin{abstract}
Fragmentation of granular clusters may be studied by experiments and by granular mechanics simulation. When comparing results, it is often assumed that results can be compared when scaled to the same value of $E / E_{\text {sep }}$, where $E$ denotes the collision energy and $E_{\text {sep }}$ is the energy needed to break every contact in the granular clusters. The ratio $E / E_{\text {sep }} \propto v^{2}$ depends on the collision velocity $v$ but not on the number of grains per cluster, $N$. We test this hypothesis using granular-mechanics simulations on silica clusters containing a few thousand grains in the velocity range where fragmentation starts. We find that a good parameter to compare different systems is given by $E /\left(N^{\alpha} E_{\mathrm{sep}}\right)$, where $\alpha \sim 2 / 3$. The occurrence of the extra factor $N^{\alpha}$ is caused by energy dissipation during the collision such that large clusters request a higher impact energy for reaching the same level of fragmentation than small clusters. Energy is dissipated during the collision mainly by normal and tangential (sliding) forces between grains. For large values of the viscoelastic friction parameter, we find smaller cluster fragmentation, since fragment velocities are smaller and allow for fragment recombination.
\end{abstract}

Keywords Cluster collisions · Granular mechanics $\cdot$ Fragmentation $\cdot$ Energy dissipation

\section{Introduction}

The understanding of the dynamics of dust particles and of agglomerates that consist of dust grains is of fundamental importance in the field of planet formation [1]. The earliest stage of the formation process is believed to be the agglomeration of dust grains in accretion disks around protostars as already discussed by Weidenschilling [2] who argues that relative velocities in such disks are sufficiently high to enable collisions, as well as by Weidenschilling and Cuzzi [3] who discuss turbulence as the driving force for particle collisions.

In such studies, the question whether agglomerates will fragment during collisions or stick and enable grain growth is of prime importance. This issue has been addressed by a variety of experimental [4-9] and theoretical/computational approaches [10-14], as reviewed in Refs. $[15,16]$. The

Herbert M. Urbassek

urbassek@rhrk.uni-kl.de

http://www.physik.uni-kl.de/urbassek/

1 Physics Department and Research Center OPTIMAS, University of Kaiserslautern, Erwin-Schrödinger-Straße, 67663 Kaiserslautern, Germany results of such studies can be used to provide basic information for use in models that describe the temporal evolution of the agglomerate size distribution in dust disks $[17,18]$.

Such models need a description of the fragmentation outcome of collisions spanning a wide range of cluster sizes and velocities, and for various relevant materials such as silica and ice. Also the comparison of experimental and computational data on cluster collisions-which have often been obtained in different cluster size regimes-needs a common base for discussion. Already early, Dominik and Tielens [10] argued that the prime quantity for cluster fragmentation in collisions is given by the ratio of the collision energy $E$ and the separation energy $E_{\text {sep }}$, which is the energy needed to break every contact in the aggregate; as soon as $E$ exceeds $E_{\text {sep }}$ by a factor of around 10, catastrophic disruption of the cluster was predicted. This argument was later used repeatedly in order to discuss experimental and computational results of cluster collisions $[4,13,19]$.

In the present paper, we analyze the validity of the argument that $E / E_{\text {sep }}$ describes the fragmentation behavior in cluster collisions using granular mechanics simulations. To this end we vary the collision energy systematically by changing either the cluster size or the cluster velocity; we also change the energy dissipation during the collision to 
obtain a greater variety of collision results under otherwise identical conditions. The results will show that $E / E_{\mathrm{sep}}$ is not a good scaling parameter to describe fragmentation. However, we will demonstrate that $E /\left(N^{\alpha} E_{\text {sep }}\right)$, where $N$ is the number of grains per cluster and $\alpha \sim 2 / 3$, describes fragmentation well, and will discuss this finding.

\section{Methods}

\subsection{Granular mechanics}

Granular mechanics is the theory describing interactions of grains of a finite size. A theoretical description of grain interactions needs to know whether or not two grains are in contact and can then derive forces and torques based on the relative motion of the grains.

We use spherical grains, where each grain has the same radius, $r$, described by the materials parameters $M$ and $\gamma$. Here $M=Y /(1-v)^{2}$ is the indentation modulus with Young's modulus $Y$ and Poisson number $v$, and $\gamma$ is the specific surface energy. The model used in our work has already been used previously [19-22], and we give a short summary here for convenience.

Let us denote grain positions by $\vec{x}$, velocities by $\vec{v}$ and angular velocities by $\vec{\omega}$. Indices $i$ and $j$ refer to the respective grains. Two grains $i$ and $j$ interact with each other if their overlap

$\delta=2 r-\left|\vec{x}_{i}-\vec{x}_{j}\right|$

is greater than 0 . The contact area of two spherical grains is a circle of radius

$a=\sqrt{\delta r / 2}$.

The relative motion can be split into normal and tangential motion as follows. With the normal unit vector

$\hat{n}=\frac{\vec{x}_{i}-\vec{x}_{j}}{\left|\vec{x}_{i}-\vec{x}_{j}\right|}$,

the contact velocity is defined as

$\vec{v}_{\mathrm{c}}=\vec{v}_{i}-\vec{v}_{j}-r\left(\vec{\omega}_{i}+\vec{\omega}_{j}\right) \times \hat{n}$.

The normal component of the velocity, $\vec{v}_{\mathrm{n}}$, is obtained by projecting $\vec{v}_{\mathrm{c}}$ on $\hat{n}$, and the tangential contribution of the velocity is then given by $\vec{v}_{\mathrm{t}}=\vec{v}-\vec{v}_{\mathrm{n}}$. The unit tangent vector is defined as

$\hat{t}=\frac{\vec{v}_{\mathrm{t}}}{\left|\vec{v}_{\mathrm{t}}\right|}$.
The normal force is given as a sum of the adhesive force and a viscoelastic term,

$F_{\mathrm{n}}=2 \pi \gamma r-\frac{2}{3} M \sqrt{\frac{r \delta}{2}}\left(\delta+A v_{\mathrm{n}}\right), \quad \delta>0$,

and vanishes for negative overlap $\delta$. The parameter $A$ in the viscoelastic term controls the amount of friction in normal motion and will be denoted as friction parameter.

In a similar way, the relative angular velocity $\vec{\omega}=\vec{\omega}_{i}-\vec{\omega}_{j}$ can be split up into two contributions, twisting (twist), and rolling (roll),

$\vec{\omega}_{\mathrm{twist}}=(\vec{\omega} \cdot \hat{n}) \hat{n}$,

$\vec{\omega}_{\text {roll }}=\vec{\omega}-\vec{\omega}_{\text {twist }}$

Tangential forces and torques for sliding (slid), rolling and twisting motion can be expressed as

$\vec{F}_{\text {slid }}=-\hat{t} \frac{Y}{4(1+v)} \pi a^{2}$,

$\vec{d}_{\text {slid }}=r \hat{n} \times \vec{F}_{\text {slid }}$,

$\vec{d}_{\text {roll }}=-4 \pi \gamma r \xi_{\text {yield }} \hat{\omega}_{\text {roll }}$

$\vec{d}_{\mathrm{twist}}=-\frac{2}{3} \frac{Y}{1+v} \frac{a^{3}}{\pi} \hat{\omega}_{\mathrm{twist}}$,

respectively, where the hat $\left(^{\wedge}\right)$ indicates a unit vector. $\xi_{\text {yield }}$ denotes the critical distance over which two spheres can roll without breaking bonds.

Further details of the algorithm are provided in Ref. [20].

\subsection{Materials parameters}

We use silica grains with a set of standard parameters $[20,23,24]$ as silica is often used in laboratory experiments as an analogue of interplanetary grain material [16, 25-27]. The grains have a radius of $r=0.76 \mu \mathrm{m}$ and a mass density of $\rho=2000 \mathrm{~kg} / \mathrm{m}^{3}$; the mass of a grain is thus $m=3.68 \cdot 10^{-15} \mathrm{~kg}$. The elastic properties are given by $M=Y /\left(1-v^{2}\right)=55.6 \mathrm{GPa}$ with Young's modulus $Y=54 \mathrm{GPa}$ and Poisson number $v=0.17$. The specific surface energy is assumed as $\gamma=0.05 \mathrm{~J} / \mathrm{m}^{2}$. We recently noted that our implementation of the granular-mechanics scheme uses only half of Young's modulus.

The parameter $A$ which controls the friction in normal motion has a standard value of $A_{\text {orig }}=0.5 \mathrm{~ns}$, as determined from experimental data [20]. We allow it here to vary between $0.5 A_{\text {orig }}$ to $1.00 A_{\text {orig }}$ in steps of $0.25 A_{\text {orig. }}$. This variation was done in order to allow for some variation in the 
fragmentation results for identical clusters (structure, $v, N$ ), as well as to study the influence of energy dissipation on the fragmentation behavior. The rolling-friction parameter $\xi_{\text {yield }}$ is set to $3.2 \mathrm{~nm}$ as recommended by Heim et al. [25].

The collision energy $E$, i.e., the kinetic energy at the start of the simulation, where 2 clusters are shot towards each other with a relative velocity $v$, can be expressed as

$E=N \frac{\pi}{3} r^{3} \rho v^{2}$,

and is thus seen to be proportional to $N$ and $v^{2}$. For a velocity of $v=10 \mathrm{~m} / \mathrm{s}$, it is $E=92.0 \cdot N \mathrm{fJ}$.

For studying fragmentation, the so-called break-up energy $E_{\text {break }}$ is introduced, which represents the energy necessary to break a contact. It is given by the work required to move two grains from their equilibrium overlap (i.e., the overlap, where adhesion and elastic repulsion cancel) to an overlap of 0 ; it is [28]

$E_{\text {break }}=K\left(\frac{\gamma^{5} r^{4}}{M^{2}}\right)^{1 / 3}$.

Here $K$ is a numerical constant equal to $K=(12 / 5)$ $(3 / 2)^{2 / 3} \pi^{5 / 3}=21.19$ [28]. In our case, it is $E_{\text {break }}=68.46$ aJ. Note that normal friction is neglected in the calculation of $E_{\text {break }}$, Eq. (14), and will lead to an increase in this quantity. While the dependence of $E_{\text {break }}$ on the materials parameters is common knowledge [10], the prefactor $K$ has been intensely discussed. Dominik and Tielens [10] advocate an increased value in order to take into account the energy dissipation by vibrations induced by the collision. From a consideration of experimental data [4], Ormel et al. [13] even argue for a value of $K=1760$. However, the magnitude of $K$ does not influence the scaling behavior of fragmentation, which is the topic of the present study.

In addition, we define the total separation energy of a cluster (break-up of all contacts) as

$E_{\text {sep }}=N_{c} E_{\text {break }}$,

where $N_{c}$ denotes the number of contacts in each of the colliding clusters.

\subsection{Simulations and their analysis}

We build spherical granular clusters of radius $R$ containing a specified number of grains, $N$, with a specified filling factor $\phi$. In this work, we use $\phi=0.28$. For the values of $N=1250,2500$ and 3750 grains, the clusters thus have radii of $R=12.51,15.77$ and $18.05 \mu \mathrm{m}$, respectively.

The clusters are constructed by an algorithm based on Ref. [21], which allows to produce clusters with pre-defined filling factor inside a spherical region. In short, new grains are added to the growing cluster at the positions of the smallest local filling factor until the prescribed number of grains have been put into the sphere. The local filling factor is calculated in a sphere of radius $3 r$ around each grain. If a new grain has been rejected 9 times_-grains are rejected if they overlap with other grains - it is attached to a randomly chosen grain; such situations may occur for large filling factors. Finally, the cluster is allowed to relax.

Since the grains are placed step by step by attaching one grain, the expected number of contacts is $N_{c}=N-1$. In fact, we find 1251, 2506 and 3754 contacts, respectively. Thus, it holds to a good approximation $N_{c}=N$. The separation energies thus amount to 85.6, 171.6 and $257.0 \mathrm{fJ}$ for clusters with $N=1250,2500$ and 3750 grains.

We study the collision of two clusters with relative velocity $v$ in the range of $10-30 \mathrm{~m} / \mathrm{s}$; the velocity is varied in steps of $5 \mathrm{~m} / \mathrm{s}$. To this purpose, we duplicate the cluster and rotate it randomly. We perform only central collisions, i.e., with a vanishing impact parameter. In order to allow for statistical reliability of the results, each simulation is repeated 10 times; in each simulation, the relative orientations of the two colliding clusters are changed randomly. The results presented below are all averages over these 10 simulations. The setup is shown schematically in Fig. 1, while Fig. 2 displays snapshots of an actual collision.

This work uses the granular mechanics code LIGGGHTS [29], which implements the forces described in Sect. 2.1 above. Every simulation is performed with $1.2 \cdot 10^{6}$ time steps of $50 \mathrm{ps}$, leading to a total duration of $60 \mu \mathrm{s}$.

To analyze the collision results, we use the algorithm of cluster analysis proposed by Stoddard [30], which identifies the fragments generated by the collision. Let us denote by $N_{1}$ and $N_{2}$ the numbers of grains in the two largest fragments. We calculate the agglomeration parameter [31],

$X=\frac{N_{1}-N_{2}}{2 N}$,

and the fragmentation parameter [31],
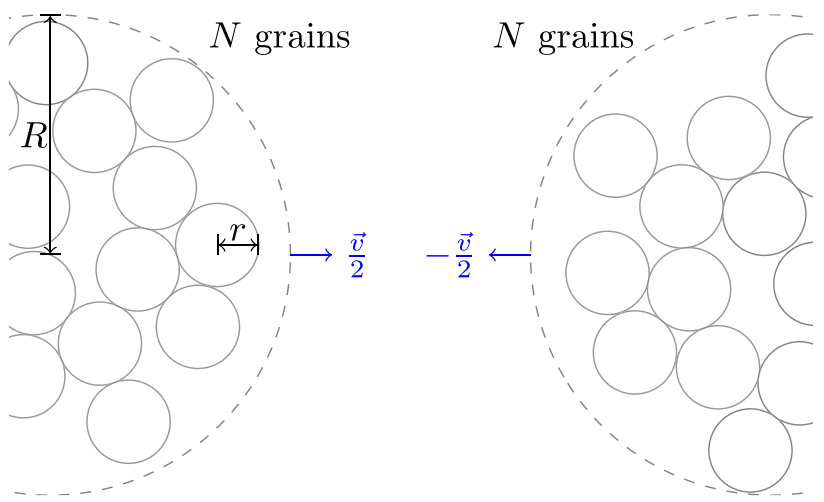

Fig. 1 Schematic setup of a cluster-cluster collision 
Fig. 2 Snapshots of a collision of two clusters with $N=3750$ grains each at $v=10 \mathrm{~m} / \mathrm{s}$ and $A=A_{\text {orig }}$, at a the beginning of the simulation, b $2 \mu \mathrm{s}, \mathbf{c} 4 \mu \mathrm{s}, \mathbf{d} 10 \mu \mathrm{s}$ and e $50 \mu \mathrm{s}$. The grains are colored according to their velocity

$N_{s}=1-\frac{N_{1}+N_{2}}{2 N}$.

It is possible to calculate the grain density by using the methods of smoothed-particle dynamics [32, 33]. For any point $\vec{r}$ in space, the grain density can be calculated as

$\rho_{\text {grains }}(\vec{r})=\sum_{i=1}^{N_{\text {grains }}} \frac{\exp \left(-\frac{1}{2}\left(\vec{r}-\vec{x}_{i}\right)^{\mathrm{T}} \Sigma^{-1}\left(\vec{r}-\vec{x}_{i}\right)\right)}{\sqrt{(2 \pi)^{3} \operatorname{det}(\Sigma)}}$.

Here the Gaussian serves as a a smoothing kernel. The matrix $\Sigma$ is a $3 \times 3$ diagonal matrix, $4 r^{2} \mathbb{1}$. Thus each grain is smeared over a width of $2 r$.

Similarly, the density of grain contacts can be calculated by substituting $\vec{x}_{i}$ in Eq. (18) by the contact points, $\vec{x}_{\mathrm{c} i}=\left(\vec{x}_{i}+\vec{x}_{j}\right) / 2$, if $i$ and $j$ are in contact, and summing over all contacts. The kinetic energy density is also given in analogy to Eq. (18), if the smoothing kernel is multiplied by the kinetic energy, $E_{i}$, of grain $i$. We determine $E_{i}$ from both the translational and rotational energy of grain $i$; however, the rotational energy is always at least a factor of $10^{-3}$ smaller than the translational energy. The kinetic energy of grain $i$ is determined relative to the center of mass of its home cluster.

\section{Results}

\subsection{Representative case: time evolution}

We show the time evolution of a prototypical collision $-N=3750, A=A_{\text {orig }}, v=10 \mathrm{~m} / \mathrm{s}$ - in the snapshots provided in Figs. 3, 4 and 5. In these snapshots, the densities-as calculated by Eq. (18) and its analogs-are plotted in a plane containing the collision axis.

In the following, we denote as the contact plane the midplane between the two clusters, perpendicular to the collision axis. Here, the two clusters meet and the most energetic processes are going to occur in its neighborhood, which we denote as the collision zone, see Fig. 5a.

The grain density, Fig. 3, shows how during the collision the grain density in the collision zone strongly increases-approximately by a factor of 2 . At around $3 \mu \mathrm{s}$, the shape of the merged cluster is almost spherical, but due to grain compression, its volume has considerably shrunk along the collision axis. In lateral direction, however, the merged cluster expanded slightly. The final

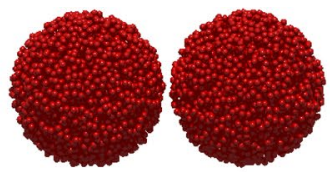

(a)

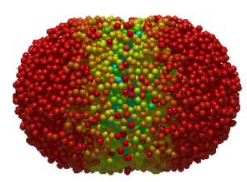

(b)

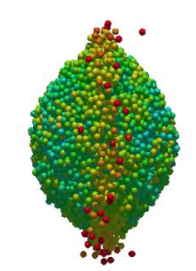

(c)

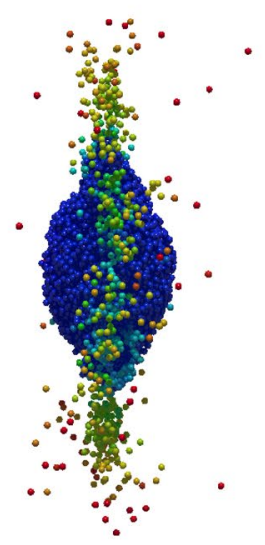

(d)

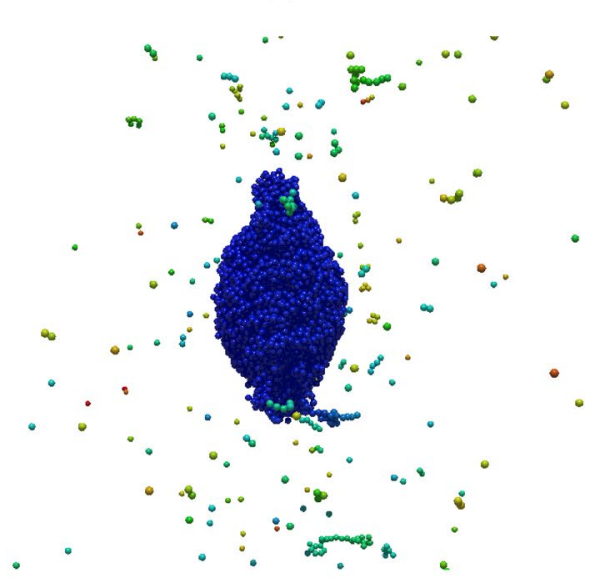

(e)

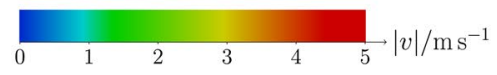


Fig. 3 Snapshots showing the grain density for a collision of two clusters $N=3750, A=A_{\text {orig }}, v=10 \mathrm{~m} / \mathrm{s}$. Data at a the start of the simulation, at b $1 \mu \mathrm{s}, \mathbf{c} 2 \mu \mathrm{s}, \mathbf{d} 3 \mu \mathrm{s}$ and $\mathbf{e} 10 \mu \mathrm{s}$

shape at the end of the collision is oblate, as the merged cluster has shrunk considerably along the collision axis.

The density of contacts, Fig. 4, shows similar features. After $1 \mu \mathrm{s}$, many contacts in the central collision region are broken. Already at $2 \mu \mathrm{s}$, the destroyed contacts in the collision zone have not only been reformed but new contacts have been generated. Note that at this time, the highly coordinated core region is surrounded by a shell of low contact density, which indicates how the collision energy spreads out of the primary collision zone and destroys other grain contacts. At $3 \mu \mathrm{s}$, the contact density in the collision zone is even higher than the grain density, while initially, it had the same magnitude. This shows that during grain compaction, the number of contacts per grain, i.e., the coordination number, has increased above the value of 2 characteristic for clusters assembled by ballistic aggregation [14]. This effect has spread over the entire merged cluster at the end of the simulation.

The energy density, Fig. 5, shows most clearly how the energy slowly diffuses out of the contact region, see Fig. $5 \mathrm{a}$ at $1 \mu \mathrm{s}$, to the entire merged cluster. At $3 \mu \mathrm{s}$, the kinetic energy is only present in a bulge surrounding the merged cluster at the periphery of the collision zone, while all other energy has been dissipated away. We do not show the energy density at the end of the simulation, since it is close to zero everywhere.

Already in this simulation, around $52 \%$ of the grains were ejected from the merged cluster; this fraction keeps increasing with collision velocity. At $30 \mathrm{~m} / \mathrm{s}$, only $3.4 \%$ of the grains are retained in the largest fragment.

We conclude that these snapshots show that the collision is terminated within approximately $3 \mu \mathrm{s}$. The clusters do not interpenetrate each other at this filling factor, 0.28 , but are stopped at the surface such that a thin collision zone develops, in which contacts are destroyed. The energy spreads out from this zone into the remaining parts of the clusters but has dissipated away at the end of the simulation. The collision zone has been compacted and grain coordination has been increased.

The results for other simulations look similar. The merged cluster becomes flattened along the collision axis for higher velocities such that it assumes a pancake shape, and more grains are ejected from it, see also Fig. 2. Further grain-based snapshots of such events can be seen in Figs. 7 and 9 of Ref. [19].

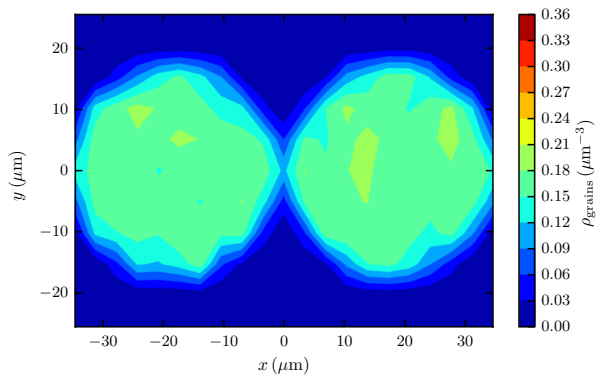

(a)

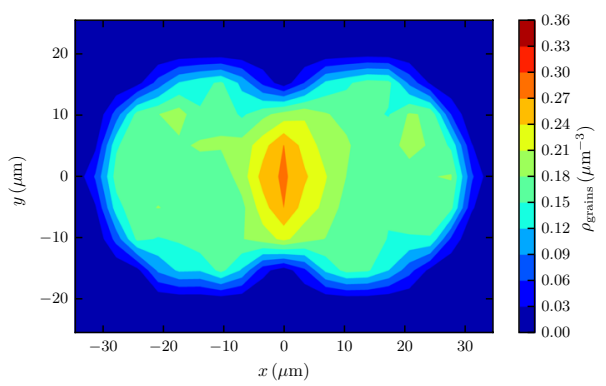

(b)

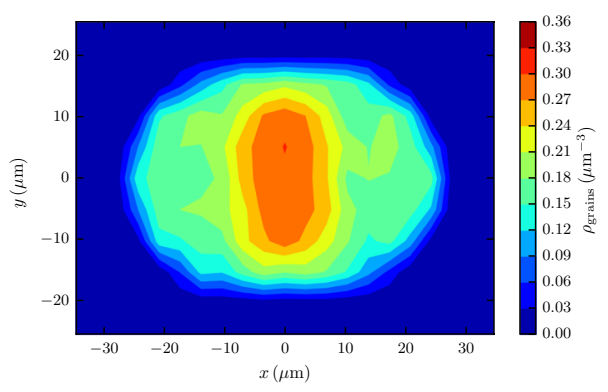

(c)

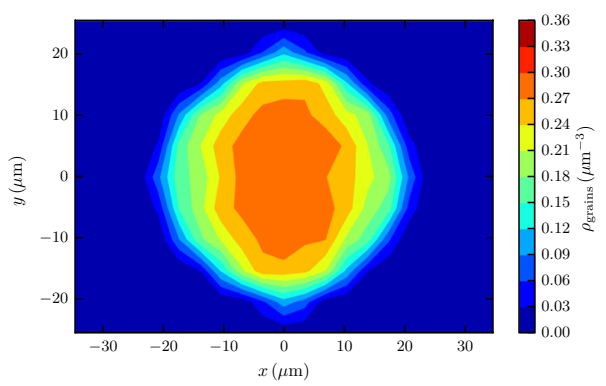

(d)

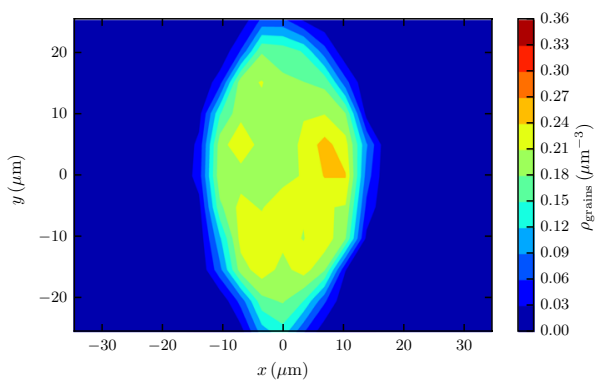

(e) 


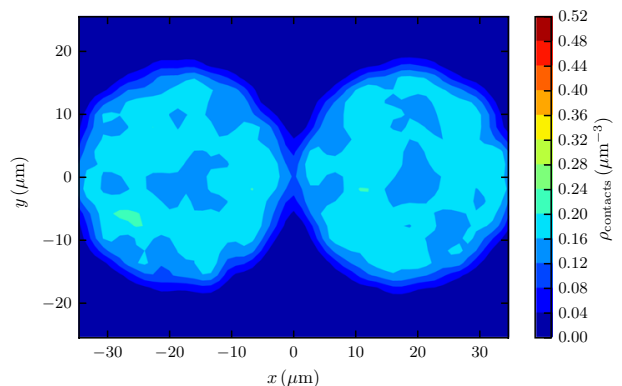

(a)

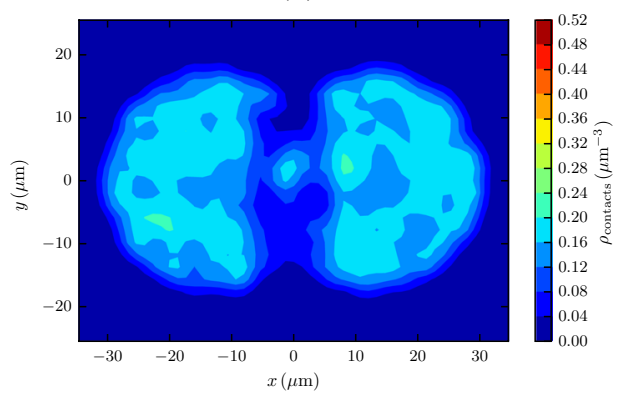

(b)

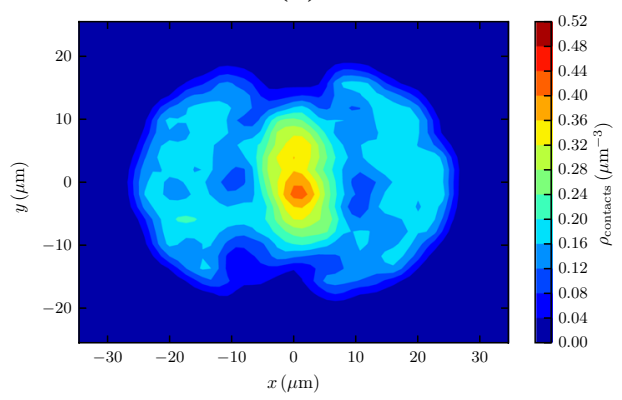

(c)

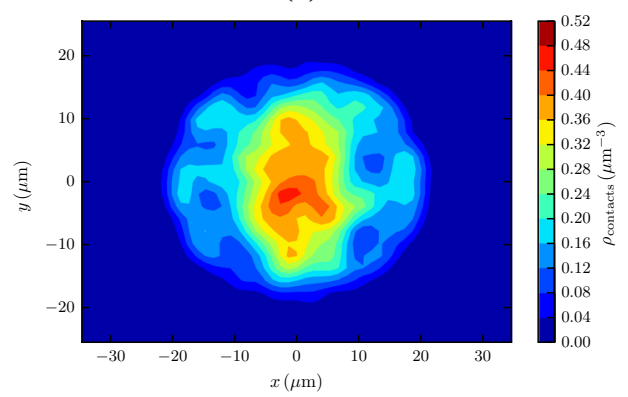

(d)

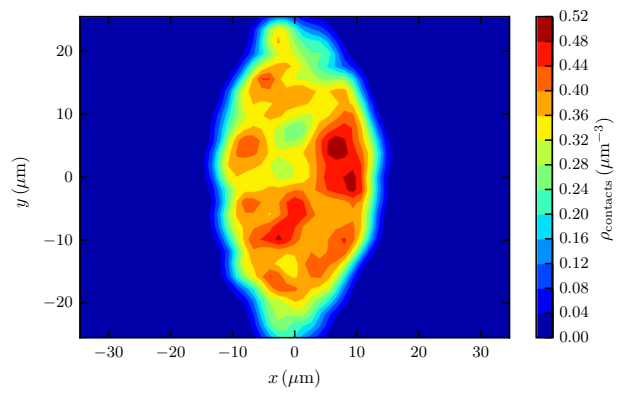

(e)

Fig. 4 Density of contacts for the collision shown in Fig. 3

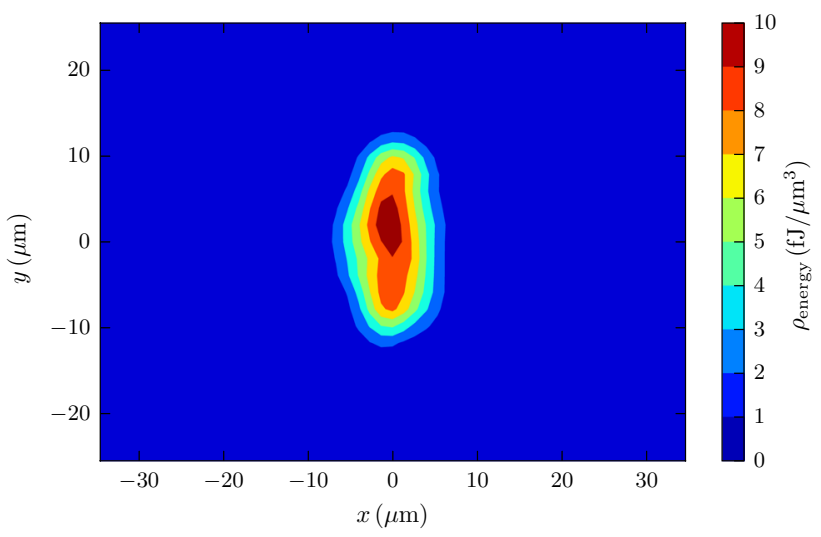

(a)

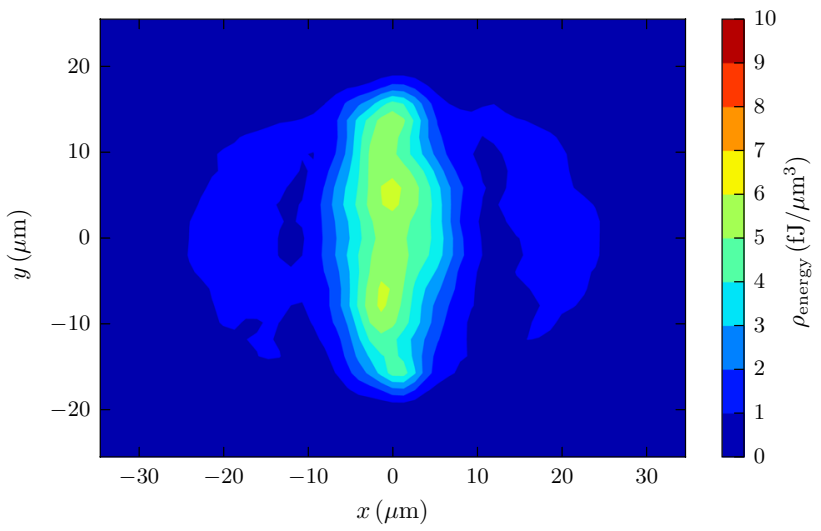

(b)

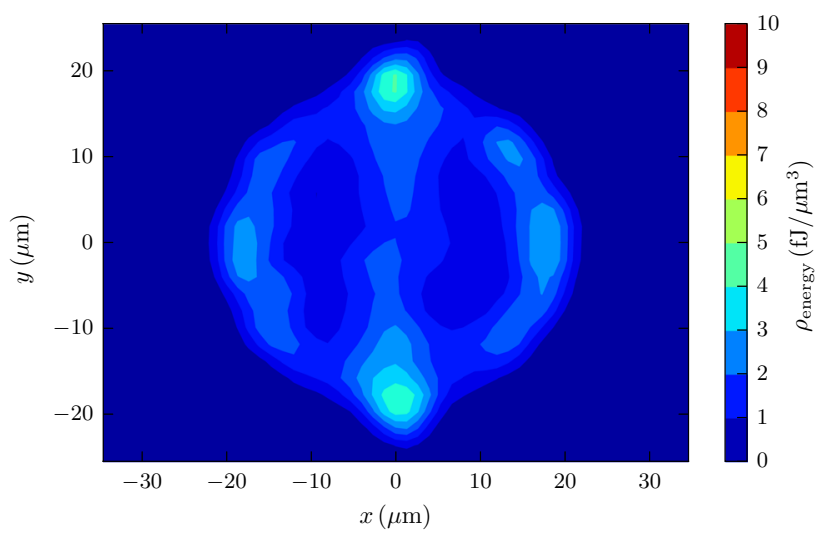

(c)

Fig. 5 Energy density for the collision shown in Fig. 3. Data at the start of the collision are not shown. Data at $\mathbf{a} 1 \mu \mathrm{s}, \mathbf{b} 2 \mu \mathrm{s}$ and $\mathbf{c} 3 \mu \mathrm{s}$

\subsection{Scaling}

The agglomeration parameter $X$, Eq. (16), is large if the largest fragment-i.e., the merged cluster-contains almost all the mass of the system. It is often assumed $[19,31]$ that small small values $X<0.15$ identify the fragmentation regime. On the other hand, the fragmentation parameter, 
$N_{s}$, Eq. (17), assumes large values when the mass contained in the two largest fragments is only a small fraction of the total mass. Hence these two parameters are often used to identify whether cluster collisions are in the sticking or in the fragmentation regime.

We present these parameters in Fig. $6 a$ and $b$ for the set of simulations performed: velocities in the range of $v=10$ $-30 \mathrm{~m} / \mathrm{s}, N=1250-3750$ grains per cluster, and friction coefficients varying between $A / A_{\text {orig }}=0.5-1.00$. The figure shows the well known facts that with increasing velocity and decreasing grain numbers, more fragmentation occurs [19, $22,34]$. In addition, we observe that with increasing dissipation (here: normal friction) fragmentation is prevented. This appears to be a novel result; it was reached here because we use exactly the same clusters at the same velocities but with changed values of the energy dissipation. It appears plausible since dissipation destroys the kinetic energy that is available for grain bouncing and hence for cluster fragmentation. The effect is astonishingly strong; by reducing the friction coefficient by a factor of 0.5 , nearly all collisions in the velocity and size window studied here become fragmenting. A reduction of $A$ by a factor of 2 has a similar-but

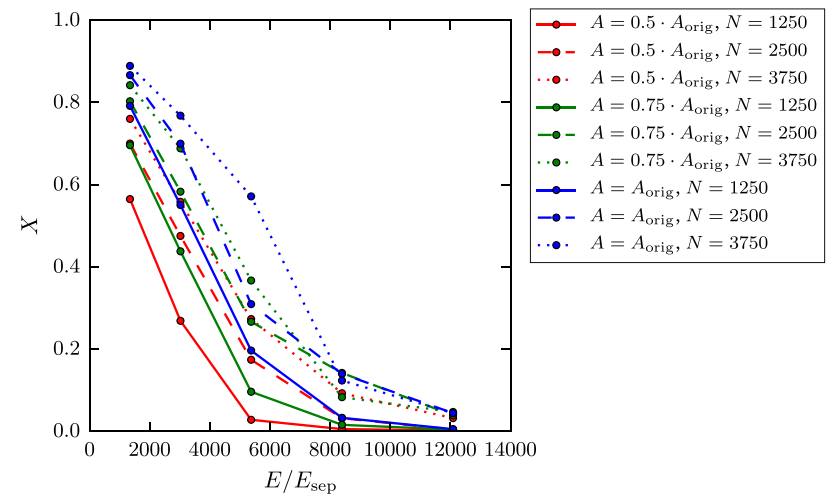

(a)

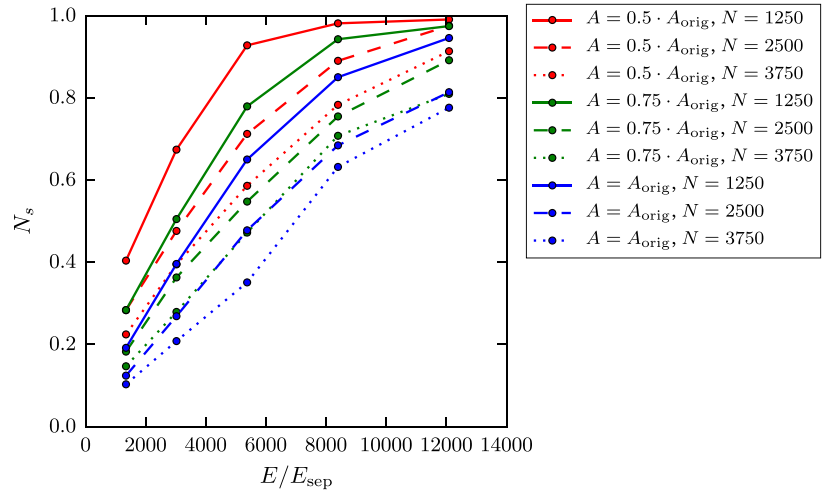

(b)

Fig. 6 a Agglomeration parameter $X$ and $\mathbf{b}$ fragmentation parameter $N_{s}$ as a function of $E / E_{\text {sep }}$ for collisions of clusters with various grain numbers, $N$, and friction coefficients, $A$ somewhat more pronounced-effect as a reduction of the collision energy by a factor of 2 . This is also the reason why we did not change the friction coefficient in a wider range.

In Fig. 6, we used the scaling parameter $E / E_{\text {sep }}$, which compares the available collision energy to the total energy needed to break all intergranular contacts, $E_{\text {sep }}=N E_{\text {break }}$, Eq. (15). For fixed $N$ and $A$, the energy is varied by the cluster velocity, $v$. Such a scaling has been proposed by Dominik and Tielens [10] and has been used repeatedly to compare fragmentation results among different clusters [4, 13, 19].

Figure 6 shows that this parameter does not allow to collapse all collision data on a single curve. However, this is possible, when changing the scaling parameter to explicitly contain the number of grains, $N$. Figure 7 shows that our data collapse on a single curve for all $N$, if we use the scaling parameter $E /\left(N^{\alpha} E_{\mathrm{sep}}\right)$; the best results were achieved with $\alpha$ in the range $0.6-0.7$. We will use $\alpha=2 / 3$ from now on. We note that for our clusters, $E / E_{\text {sep }} \propto v^{2}$, since the grain number drops out from the ratio, and the materials parameters appearing in $E_{\text {break }}$ are unchanged. A scaling parameter $E /\left(N^{\alpha} E_{\text {sep }}\right)$ with $\alpha \neq 0$ thus means that cluster size explicitly enters the description.

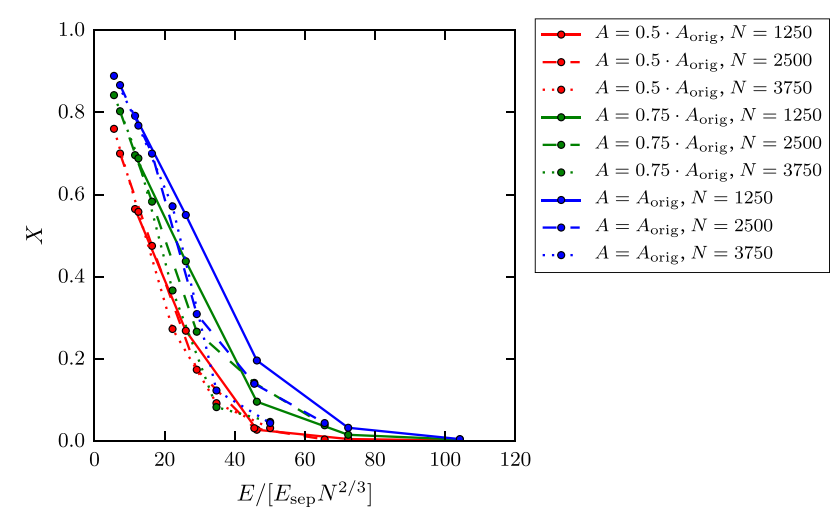

(a)

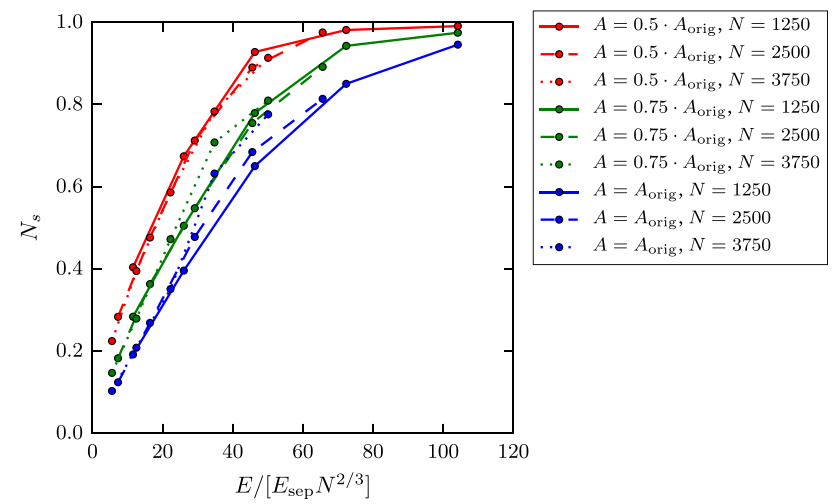

(b)

Fig. 7 Same data as in Fig. 6, but plotted versus $E /\left[E_{\text {sep }}\left(2 N_{c}\right)^{2 / 3}\right]$ 
Data collapse works better with the fragmentation parameter $N_{s}$ than with the agglomeration parameter $X$. This finding coincides with our experience that $X$ has some ambiguity in defining fragmentation: small values of $X$ might either mean strong fragmentation or intact bouncing of the clusters. We therefore propose that the use of $N_{s}$ is more adequate in quantifying cluster fragmentation.

Of course, for different dissipation constants $A$, different master curves are obtained, since the details of the collision and of the fragmentation change, see Sect. 3.4. It is possible to collapse all data to just one master curve by using a parameter $E /\left[N^{\alpha} E_{\text {sep }}\left(A / A_{\text {orig }}\right]^{\beta}\right)$; Fig. 8 demonstrates that such a data collapse works well with $\beta=0.8$. The dependence on $A$ means that for lower friction, the cluster will exhibit stronger fragmentation, which is expected.

Since the number of grains scales like the cluster volume, $N \propto R^{3}$, the value of $\alpha=2 / 3$ can be associated with a cluster-size dependence corresponding to that of the cluster cross section. A value of $\alpha=2 / 3$ can, however, also be discussed in terms of the cluster passage time

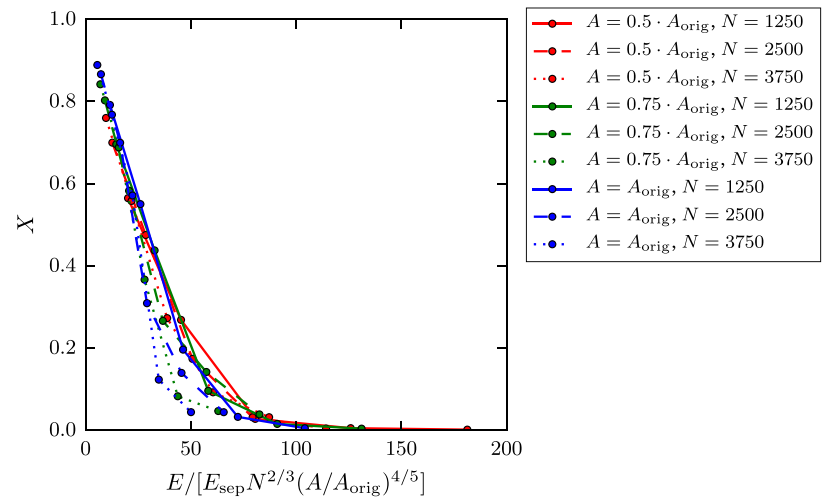

(a)

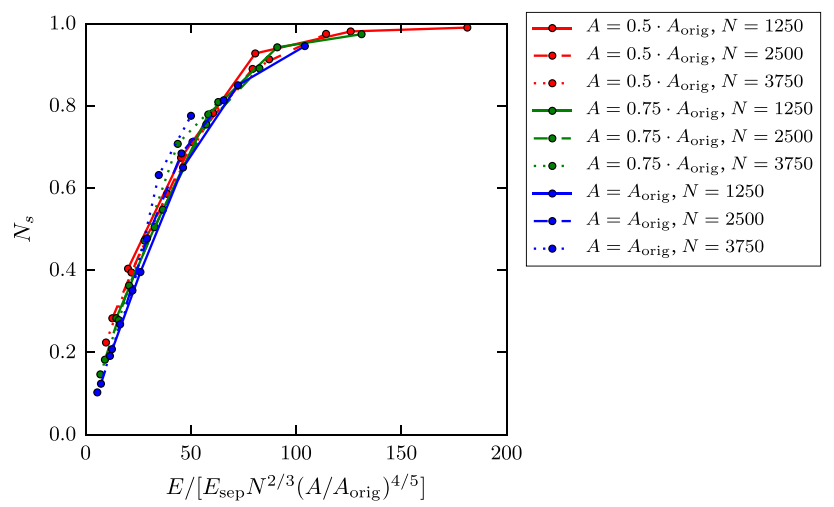

(b)

Fig. 8 Same data as in Fig. 6, but plotted versus $E /\left[E_{\text {sep }}\left(2 N_{c}\right)^{2 / 3}\right.$ $\left.\left(A / A_{\text {orig }}\right)^{0.8}\right]$ $t_{\text {pass }}=R / v$,

since then the scaling parameter $E /\left(N^{2 / 3} E_{\text {sep }}\right) \propto(\nu / R)^{2}=t_{\text {pass. }}^{-2}$.

Our result that the relevant scaling parameter for cluster fragmentation in collisions, $E /\left(N^{\alpha} E_{\text {sep }}\right)$ with $\alpha=2 / 3$, thus points at the fact that during cluster stopping in the collision, the collision energy cannot be used directly to break all clusters. Fragmentation first occurs in the shallow collision zone with an area $\propto N^{2 / 3}$ and then expands into the entire merged clusters (volume $\propto N$ ). These features explain the exponent of $\alpha=2 / 3$. Evidently, energy dissipation in the collision dictates to what extent the entire collision energy is available to break all bonds, or is dissipated away, before it can reach each grain. For this reason, we now discuss the role of energy dissipation in the collisions studied here.

\subsection{Time dependence of fragmentation}

Figure $9 \mathrm{a}$ and $\mathrm{b}$ show the fragmentation parameter plotted as a function of time for two collisions of clusters of $N=1250$ grains each at collision velocity $v=20 \mathrm{~m} / \mathrm{s}$, with friction parameters $A=0.5 A_{\text {orig }}$ and $A=A_{\text {orig }}$. We observe that for both collisions, $N_{s}$ goes up to almost 1 at times of 1.5-2.5 $\mu$ s, indicating complete fragmentation. However, at later times, $N_{s}$ decreases again; this means that grains assemble again to form clusters. This process is particularly pronounced for the higher value of the friction parameter, Fig. 9b. The ensuing decrease in $N_{s}$ shows that fragmentation is not a simple process of breaking up several contacts just once. In fact, contacts can break, but contacts can also form (temporarily as well as permanently). In this specific case, contacts are more likely to form again for higher friction, since higher friction dissipates more kinetic energy and grains collide with lower relative velocities allowing for more new contacts to form. This is not only influenced by friction, but by the initial collision velocity as well, see Fig. 9c, since at lower collision velocities, contacts can form again.

\subsection{Energy dissipation}

For studying energy dissipation, we determine the time dependence of the power lost in the collision by all dissipation channels (sliding, twisting, rolling, normal motion). The power lost by dissipative forces is given by

$P_{\mathrm{fs}}(t)=\sum_{i=1}^{N} \sum_{j \neq i} \vec{F}_{\mathrm{s}}^{i j} \cdot \vec{v}_{i}$,

and the power lost by dissipative torques is given by 


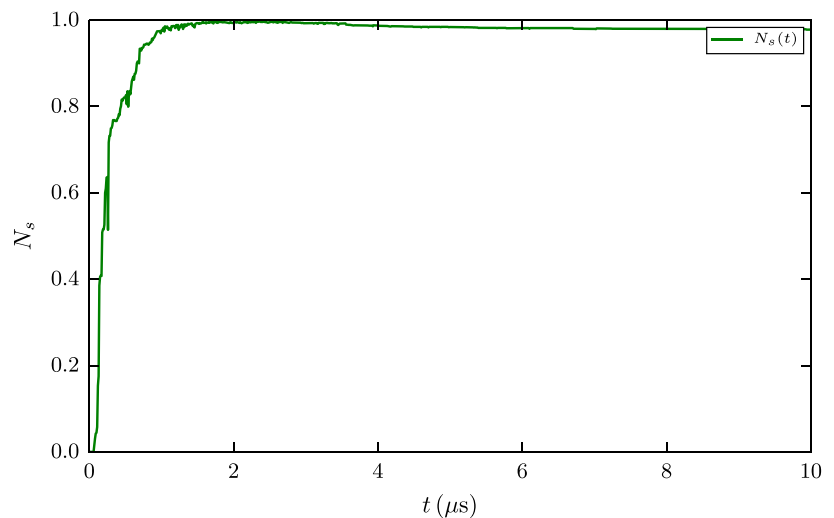

(a)

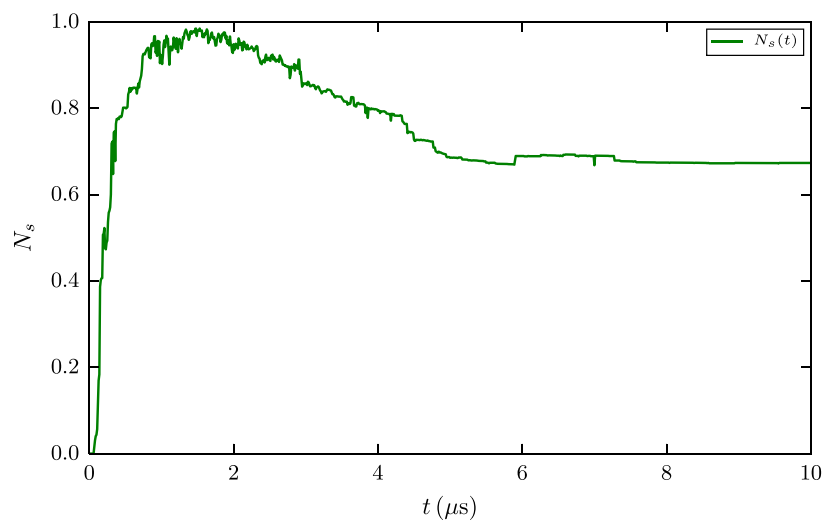

(b)

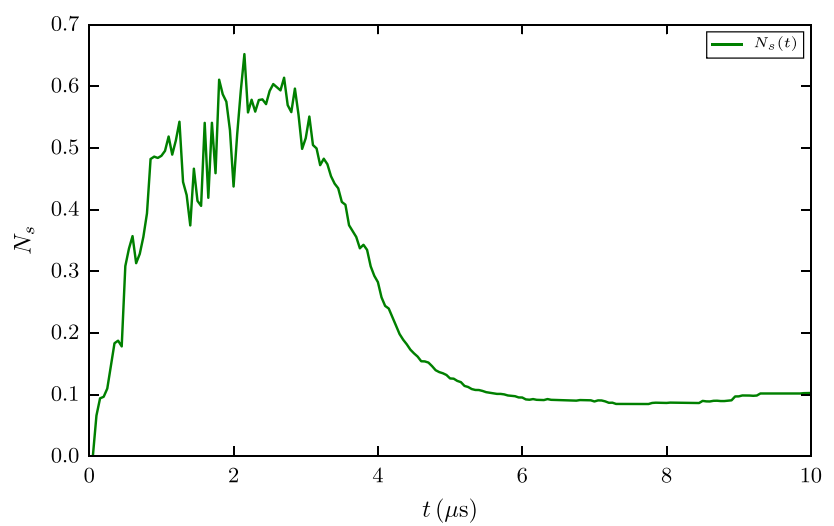

(c)

Fig. 9 Temporal evolution of the fragmentation parameter, $N_{s}$, for a collision of clusters with $N=1250$ grains each, at $v=20 \mathrm{~m} / \mathrm{s}$ for friction parameters of $\mathbf{a} A=0.5 A_{\text {orig }}$ and $\mathbf{b} A=A_{\text {orig. }}$. c Shows this quantity for large clusters, $N=3750$, at lower velocity, $v=10 \mathrm{~m} / \mathrm{s}$ and with friction parameter $A=A_{\text {orig }}$

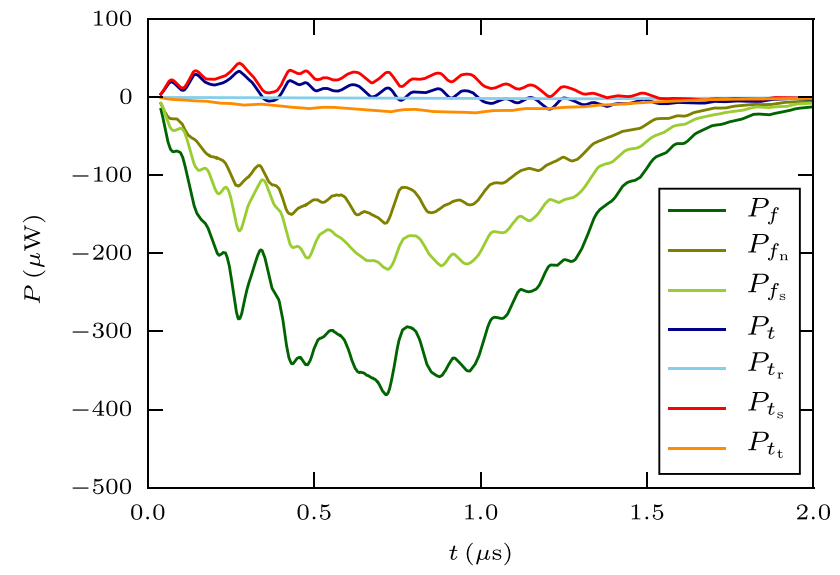

(a)

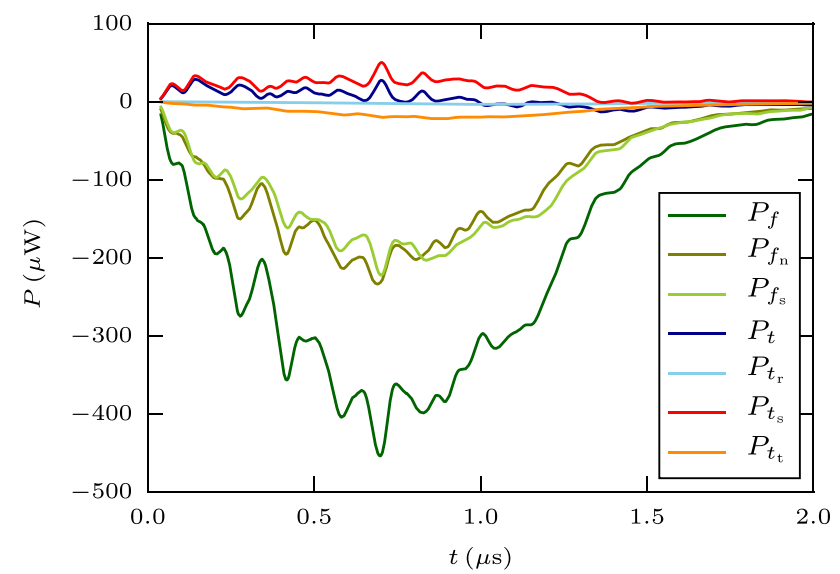

(b)

Fig. 10 Temporal evolution of the power dissipated in the various dissipation channels for a collision of clusters with $N=1250$ grains per cluster at a collision velocity of $v=20 \mathrm{~m} / \mathrm{s}$, for friction parameters of $\mathbf{a} A=0.5 A_{\text {orig }}$ and $\mathbf{b} A=A_{\text {orig }}$

$P_{\mathrm{ts}}(t)=\sum_{i=1}^{N} \sum_{j \neq i} \vec{d}_{\mathrm{s}}^{i j} \cdot \vec{\omega}_{i}$

where we sum over forces and torques exerted by grain $j$ on grain $i$. In Fig. 10 we plot the time evolution of these powers (smoothed with a Gaussian filter of $15 \mathrm{~ns}$ width) for collisions of clusters with $N=1250$ grains per cluster at a collision velocity of $v=20 \mathrm{~m} / \mathrm{s}$ for two friction parameters, $A=0.5 A_{\text {orig }}$ and $A=A_{\text {orig. }}$. The figure shows that energy dissipation by forces (denoted by $P_{\mathrm{f}}$ ) is dominant over energy dissipation by torques $\left(P_{\mathrm{t}}\right)$. Energy dissipation due to normal-force dissipation $\left(P_{\mathrm{fn}}\right)$ and sliding-force dissipation $\left(P_{\mathrm{fs}}\right)$ is of similar order of magnitude. Since initially, the grains do not rotate, the collision excites grain rotation as can be seen by the positive sign in the sliding $\left(P_{\mathrm{ts}}\right)$ rotational mode. The twisting mode $\left(P_{\mathrm{tt}}\right)$ as well as (to a lesser extent) the rolling mode $\left(P_{\mathrm{tr}}\right)$ are de-excited. 
Table 1 Energy balance of the collision analyzed in Fig. 11 for two values of the friction constant $A$

\begin{tabular}{|c|c|c|c|c|c|c|}
\hline$A$ & $f_{\mathrm{n}}(\mathrm{pJ})$ & $f_{\mathrm{s}}(\mathrm{pJ})$ & $t_{\mathrm{r}}(\mathrm{pJ})$ & $t_{\mathrm{s}}(\mathrm{pJ})$ & $t_{\mathrm{t}}(\mathrm{pJ})$ & sum $(\mathrm{pJ})$ \\
\hline$A_{\text {orig }}$ & -192.83 & -190.28 & -4.03 & 28.63 & -20.48 & -378.99 \\
\hline $0.5 A_{\text {orig }}$ & -147.11 & -215.04 & -2.22 & 25.60 & -18.64 & -357.40 \\
\hline$A$ & $E(\mathrm{pJ})$ & $E_{\text {kin }}(\mathrm{pJ})$ & $E_{\mathrm{rot}}(\mathrm{pJ})$ & $E_{\text {diss }}(\mathrm{pJ})$ & $E_{\text {fin }}(\mathrm{pJ})$ & \\
\hline$A_{\text {orig }}$ & 459.69 & 78.67 & 3.20 & 378.99 & 460.86 & \\
\hline $0.5 A_{\text {orig }}$ & 459.69 & 98.76 & 4.04 & 357.40 & 460.20 & \\
\hline
\end{tabular}

Top: Energies dissipated within $2 \mu$ s in the various channels, and their sum. Bottom: Comparison of the collision energy $E$ with the kinetic and rotational energies of grains, $E_{\mathrm{kin}}$ and $E_{\mathrm{rot}}$, respectively, at $2 \mu \mathrm{s}$, the total dissipated energy, $E_{\mathrm{diss}}$, which is given by the sum value of (a), and the final energy, $E_{\text {fin }}=E_{\text {kin }}+E_{\text {rot }}+E_{\text {diss }}$

Table 1 assembles the energy balance at a time of $2 \mu \mathrm{s}$ quantitatively. As Fig. 10 showed, dissipation data at $2 \mu \mathrm{s}$ after the start of the collision is close to final. Table $1 \mathrm{a}$ quantifies that the energies dissipated by normal and sliding forces are dominant, while energies in rotational motion are minor; sliding induces rotation, and so this channel carries another sign than the other channels.

We compare the collision energy $E=459.7 \mathrm{pJ}$ in Table $1 \mathrm{~b}$ with the final energies. Around $20 \%$ of the initial energy are found at $2 \mu \mathrm{s}$ in translational motion of grains while their rotation carries only a minor fraction of energy. If we sum these kinetic energies with the dissipated energy, we obtain a value which agrees within better than $1 \%$ with the initial collision energy $E$. The small error is caused by the numerical integration of the power over time. The energy needed for the fragmentation of all grains, $E_{\text {sep }}$, amounts to only $85.6 \mathrm{fJ}$ in this case, and can be ignored in this calculation.

Fragment kinetic energies are higher for $A=0.5 A_{\text {orig }}$, since less energy has been dissipated, while a large number of fragments has been created which then leave the collision zone without further interaction.

One can see that for the original value of $A=A_{\text {orig, }}$, the powers dissipated through normal and sliding motion are very close to each other, while the importance of normal motion for the dissipation decreases for lower $A$. This is clear from the fact that $A$ enters into the normal force as a parameter.

A reduction of $A$ by $50 \%$ does not lead to a similar reduction in the power dissipated in the normal-force channel $\left(P_{\mathrm{fn}}\right)$, which is only reduced by $24 \%$. In addition, this reduction is partly compensated by an increase in the power dissipated by sliding motion; this increase may be induced by the longer-lived contacts (reduced fragmentation, see Fig. 9) for large $A$. As a summary, we see that a reduction of $A$ slightly (by $6 \%$ ) reduces the energy dissipation during the collision, enhancing the kinetic energy going into the fragments.

Figure 10 shows that the time scale for energy dissipation is around $2 \mu \mathrm{s}$. We may compare this time scale with the cluster passage time defined in Eq. (19). For a velocity of $v=20 \mathrm{~m} / \mathrm{s}, t_{\text {pass }}=0.63(0.79,0.9) \mu$ s for cluster sizes of
$N=1250(2500,3750)$ grains. A comparison with Fig. 10 shows that the collisions are indeed over after around (2-3) $t_{\text {pass. }}$. Such short time scales for collisions are in agreement with previous results of cluster stopping in a granular environment [35], and can be justified by referring to energyproportional stopping as in Poncelet's law [36, 37].

Figure 11 shows plots of the time evolution of the kinetic energy during the collision, averaged over 10 collisions. In Fig. 11a, we observe that for higher collision velocities, energy will initially be dissipated faster-roughly in agreement with the passage-time scale, Eq. (19)—but collisions at lower velocities dissipate energy for a longer time and, most strikingly, at the end of the simulation have dissipated a higher fraction of their starting energy. Figure $11 \mathrm{~b}$ shows a similar effect for varied cluster size, $N$, such that largecluster collisions lead to longer-lasting energy dissipation. We argue that this feature is consistent with our observation that $E / E_{\mathrm{sep}}$ is not a good parameter to describe the collisions. As the number of grains and contacts effectively cancel out of $E / E_{\text {sep }}$, this parameter does not show any dependence on cluster size. It would describe a fragmentation process well, where impact energy is distributed through the cluster instantaneously. Realistic collisions, however, will initially store their energy in the collision zone and from there energy will gradually spread throughout the cluster. If contacts break too rapidly, there is no effective means for transporting the impact energy throughout the entire clusters, and contacts far away from the impact site are not affected by the collision. Collisions at lower velocities store less energy in the collision zone, break up less contacts and allow for energy to be distributed better throughout the cluster. This allows for more energy to be dissipated eventually.

The time evolution of the energy dissipation of colliding clusters is only negligibly affected by the dissipation constant $A$, Fig. $11 \mathrm{c}$. This demonstrates that our choice of varying $A$ to allow for a larger variety of collision situations affects the collision time scale only negligibly. However, energy dissipation proceeds for longer time scales for large values of $A$. This is caused by less fragmentation 


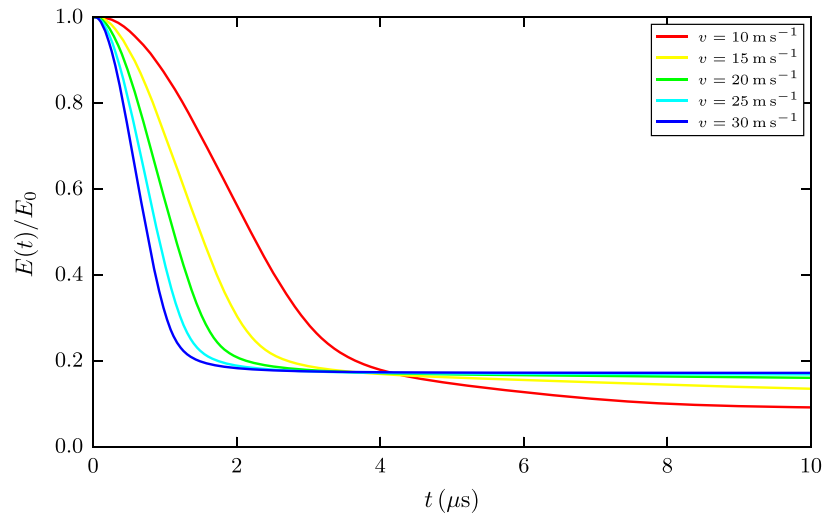

(a)

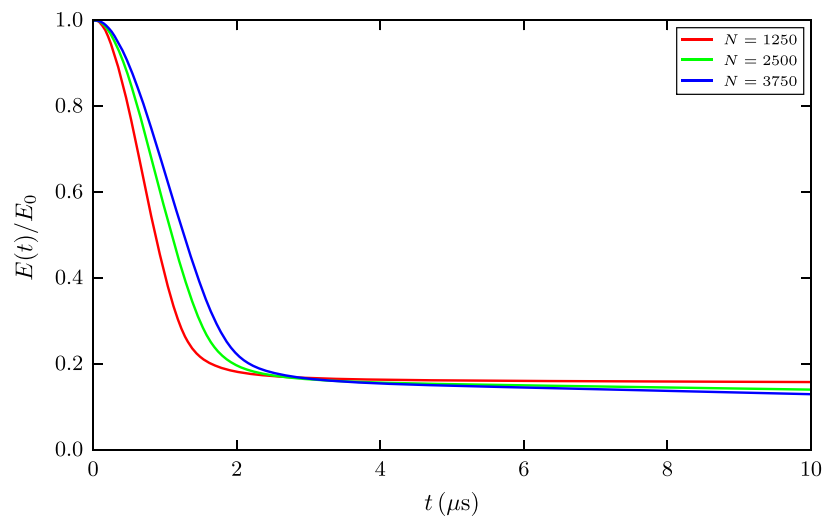

(b)

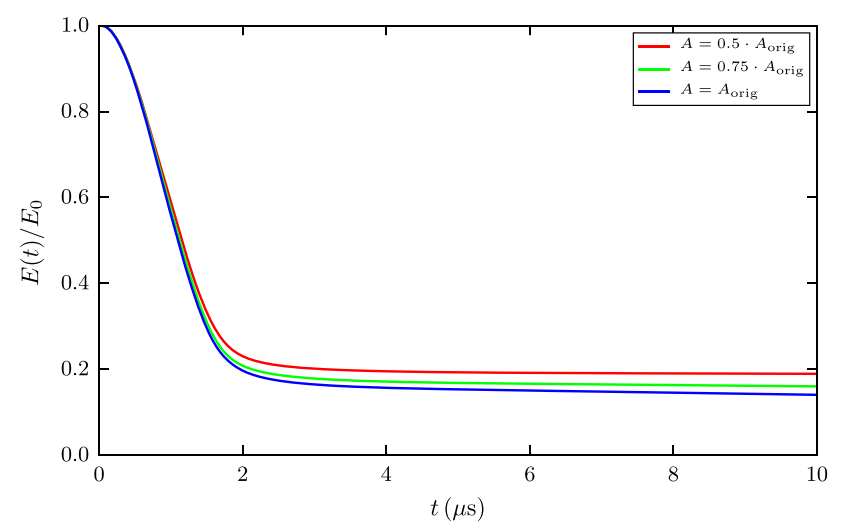

(c)

Fig. 11 Temporal evolution of the normalized kinetic energy for collisions of a clusters with $N=2500$ grains per cluster and a friction parameter of $A=0.75 A_{\text {orig }}$ at various velocities $v, \mathbf{b}$ clusters with velocity $20 \mathrm{~m} / \mathrm{s}$ and a friction parameter of $A=A_{\text {orig }}$ for various cluster sizes $N$, and c clusters with $N=2500$ grains colliding with velocity $20 \mathrm{~m} / \mathrm{s}$ for various friction parameters $A$

occurring in this case, as discussed in connection with Fig. 9.

Note that even though the energy dissipation processes do not appear to have completely stopped at the end of the simulation, the fragmentation patterns are final, see Fig. 9.
Minor relative motion in the grains still exists, but contacts do no longer change significantly.

\section{Conclusions}

We discuss the scaling of fragmentation in granular cluster collisions using as a basis a series of simulations for granular silica clusters, $\phi=0.28$, containing several thousand grains at velocities where fragmentation starts. We obtain the following findings.

1. The parameter $E / E_{\text {sep }}$ is not a good quantity to describe fragmentation. Results do not collapse on a single curve when described with $E / E_{\text {sep. }}$. This finding is in contrast to previous arguments [10].

2. However, when plotting fragmentation parameters vs. $E /\left(N^{\alpha} E_{\text {sep }}\right)$ with $\alpha=2 / 3$, data for various $N$ and $v$ collapse to a single curve.

3. We argue that the reason for the scaling with $E /\left(N^{\alpha} E_{\text {sep }}\right)$ lies in the space dependence of energy deposition during cluster collisions and in the ensuing energy dissipation. For vanishing energy dissipation, the entire collision energy $E$ could be used for fragmentation, and $\alpha=0$ would result. Taking dissipation into account, it is not the entire energy $E$ that can be used for fragmentation; rather it is the areal energy density $E / R^{2} \propto E / N^{2 / 3}$ that is relevant, since collisions initially occur in the collision plane. The energy is transported only slowly away from the collision zone while energy already dissipates away. This explains a value of $\alpha$ close to 2/3.

Cluster fragmentation is accompanied by energy dissipation. Here the potential energy spent in cluster break-up plays only a minor role in the examples studied here, since the parameter $E / E_{\mathrm{sep}}$, which describes the ratio of the available collision energy to the energy needed to fragment the cluster completely, is of the order of 1000 . We find that the collision energy is spent mostly by normal and tangential (sliding) forces between grains, while torques mediated by grain rotation are negligible in this respect.

In order to understand the effect of energy dissipation on cluster fragmentation in more detail, we allowed the viscoelastic friction parameter $A$, which describes energy dissipation of the normal contact force, to vary. Besides the obvious effect that lower values of $A$ lead to less energy dissipation and hence a higher remaining kinetic energy of the fragments, we also find that it induces higher fragmentation. This effect is counter-intuitive at first sight, but is caused by the fact that high energy dissipation leads to smaller velocities of the fragments that thus have a higher chance to recombine to clusters. Indeed we see that for high $A$, fragmentation has a non-monotonic time evolution, 
and thus features a prominent grain recombination regime. As a consequence, dissipation also lasts for a longer time than for small $A$ where the cluster is more completely fragmented.

The time scale of energy dissipation amounts to around three times the cluster passage time, Eq. (19). However, changes in the fragmentation parameters can proceed for a longer time, in particular for large clusters and low velocities, as at low energies new contacts are able to form.

In our systems, the difference between the number of grains, $N$, and the number of contacts, $N_{c}$, is negligible, since for clusters assembled by ballistic deposition, the local coordination of each grain is 2-except at the surface-such there is only a constant factor between $N$ and $N_{c}$, which is of no consequence for scaling arguments. Also for more compact clusters, where the coordination number is larger, the difference between $N$ and $N_{c}$ will only introduce a constant factor in the scaling parameter.

One might speculate to what extent the cluster shape influences collisions and the resulting fragmentation patterns. Such an influence is indeed to be expected: If two oblate clusters meet with their flat sides, more fragmentation is to be expected than for two prolate clusters colliding with their tips. This argument follows the dependence of our scaling on the cluster cross section via $N^{\alpha}$. For the dissipation of non-collisional energy input-such as by granular temperature or release of elastic energy - such an argument is not so easily available, as such an energy input might either be homogeneous in the entire cluster or follow a law not determined by the cluster cross section. We therefore do not expect our analysis to be easily extended to non-collisional energy transfers.

The fragmentation behavior during cluster collisions is relevant for instance in the field of dust agglomeration in protoplanetary disks, and our material parameters were chosen to apply for such situations. Here, scaling parameters are used for comparing experimental and simulational data, or for extrapolating available data to other size or velocity regimes. We expect that our argument that $E /\left(N^{\alpha} E_{\text {sep }}\right)$ rather than $E / E_{\text {sep }}$ is the appropriate scaling parameter for cluster fragmentation will prove useful in data comparison and extrapolation as well as in numerical schemes for calculating the temporal evolution of dust disks.

Acknowledgements Simulations were performed at the High Performance Cluster Elwetritsch (RHRK, TU Kaiserslautern, Germany).

Funding Open Access funding enabled and organized by Projekt DEAL.

\section{Declaration}

Conflict of interest The authors declare that they have no conflict of interest.
Open Access This article is licensed under a Creative Commons Attribution 4.0 International License, which permits use, sharing, adaptation, distribution and reproduction in any medium or format, as long as you give appropriate credit to the original author(s) and the source, provide a link to the Creative Commons licence, and indicate if changes were made. The images or other third party material in this article are included in the article's Creative Commons licence, unless indicated otherwise in a credit line to the material. If material is not included in the article's Creative Commons licence and your intended use is not permitted by statutory regulation or exceeds the permitted use, you will need to obtain permission directly from the copyright holder. To view a copy of this licence, visit http://creativecommons.org/licenses/by/4.0/.

\section{References}

1. Armitage, P.J.: Astrophysics of Planet Formation. Cambridge University Press, New York (2010)

2. Weidenschilling, S.J.: Aerodynamics of solid bodies in the solar nebula. Mon. Not. R. Astron. Soc. 180, 57 (1977)

3. Weidenschilling, S.J., Cuzzi, J.N.: Formation of planetesimals in the solar nebula. In: Levy, E.H., Lunine, J.I. (eds.) Protostars and Planets III, vol. 3, p. 1031. The University of Arizona Press, Tuscon (1993)

4. Blum, J., Wurm, G.: Experiments on sticking, restructuring, and fragmentation of preplanetary dust aggregates. Icarus 143, 138 $146(2000)$

5. Schräpler, R., Blum, J., Seizinger, A., Kley, W.: The physics of protoplanetesimal dust agglomerates VII. The low-velocity collision behavior of large dust agglomerates. Astron. Astrophys. 758, 35 (2012)

6. Whizin, A.D., Blum, J., Colwell, J.E.: The physics of protoplanetesimal dust agglomerates VIII Microgravity collisions between porous $\mathrm{SiO}_{2}$ aggregates and loosely bound agglomerates. Astrophys. J. 836, 94 (2017)

7. Katsuragi, H., Blum, J.: Impact-induced energy transfer and dissipation in granular clusters under microgravity conditions. Phys. Rev. Lett. 121, 208001 (2018)

8. Ellerbroek, L.E., Gundlach, B., Landeck, A., Dominik, C., Blum, J., Merouane, S., Hilchenbach, M., Bentley, M.S., Mannel, T., John, H., van Veen, H.A.: The footprint of cometary dust analogues I. Laboratory experiments of low-velocity impacts and comparison with Rosetta data. Mon. Notices R. Astron. Soc. 469, S204-S216 (2017)

9. Schräpler, R., Blum, J., Krijt, S., Raabe, J.-H.: The physics of protoplanetary dust agglomerates. X High-velocity collisions between small and large dust agglomerates as a growth barrier. Astrophys. J. 853, 74 (2018)

10. Dominik, C., Tielens, A.G.G.M.: The physics of dust coagulation and the structure of dust aggregates in space. Astrophys. J. 480, 647-673 (1997)

11. Suyama, T.: Wada, Koji, Tanaka, Hidekazu: Numerical simulation of density evolution of dust aggregates in protoplanetary disks. I. Head-on collisions. Astrophys. J. 684, 1310 (2008)

12. Wada, K., Tanaka, H., Suyama, T., Kimura, H., Yamamoto, T.: Numerical simulation of dust aggregate collisions. II. Compression and disruption of three-dimensional aggregates in head-on collisions. Astrophys. J. 677, 1296 (2008)

13. Ormel, C.W., Paszun, D., Dominik, C., Tielens, A.G.G.M.: Dust coagulation and fragmentation in molecular clouds I. How collisions between dust aggregates alter the dust size distribution. Astron. Astrophys. 502, 845 (2009)

14. Wada, K., Tanaka, H., Suyama, T., Kimura, H., Yamamoto, T.: The rebound condition of dust aggregates revealed by numerical simulation of their collisions. Astrophys. J. 737, 36 (2011) 
15. Dominik, C., Blum, J., Cuzzi, J.N., Wurm, G.: Growth of dust as the initial step toward planet formation. In: Reipurth, B., Jewitt, D., Keil, K. (eds.) Protostars and Planets V, pp. 783-800. University of Arizona Press, Tucson (2007)

16. Blum, J.: Dust growth in protoplanetary disks: a comprehensive experimental/theoretical approach. Res. Astron. Astrophys. 10, $1199(2010)$

17. Birnstiel, T., Dullemond, C.P., Brauer, F.: Gas- and dust evolution in protoplanetary disks. Astron. Astrophys. 513, A79 (2010)

18. Birnstiel, T., Ricci, L., Trotta, F., Dullemond, C.P., Natta, A., Testi, L., Dominik, C., Henning, T., Ormel, C.W., Zsom, A.: Testing the theory of grain growth and fragmentation by millimeter observations of protoplanetary disks. Astron. Astrophys. 516, L14 (2010)

19. Ringl, C., Bringa, E.M., Bertoldi, D.S., Urbassek, H.M.: Collisions of porous clusters: a granular-mechanics study of compaction and fragmentation. Astrophys. J. 752, 151-163 (2012)

20. Ringl, C., Urbassek, H.M.: A LAMMPS implementation of granular mechanics: inclusion of adhesive and microscopic friction forces. Comput. Phys. Commun. 183, 986-992 (2012)

21. Ringl, C., Bringa, E.M., Herbert, M.: Impact on porous targets: penetration, crater formation, target compaction, and ejection. Phys. Rev. E 86, 061313 (2012)

22. Gunkelmann, N., Ringl, C., Urbassek, H.M.: Influence of porosity on collisions between dust aggregates. Astron. Astrophys. 589, A30 (2016)

23. Chokshi, A., Tielens, A.G.G.M., Hollenbach, D.: Dust coagulation. Astrophys. J. 407, 806-819 (1993)

24. Blum, J., Schräpler, R.: Structure and mechanical properties of high-porosity macroscopic agglomerates formed by random ballistic deposition. Phys. Rev. Lett. 93, 115503 (2004)

25. Heim, L.-O., Blum, J., Preuss, M., Butt, H.-J.: Adhesion and friction forces between spherical micrometer-sized particles. Phys. Rev. Lett. 83, 3328-3331 (1999)

26. Blum, J., Schräpler, R., Davidsson, B.J.R., Trigo-Rodriguez, J.M.: The physics of protoplanetesimal dust agglomerates. I.
Mechanical properties and relations to primitive bodies in the solar system. Astrophys. J. 652, 1768 (2006)

27. Blum, J.: Dust evolution in protoplanetary discs and the formation of planetesimals. Space Sci. Rev. 214, 52 (2018)

28. Umstätter, P., Urbassek, H.M.: Fragmentation and energy dissipation in collisions of polydisperse granular clusters. A\&A 633, A24 (2020)

29. Kloss, C., Goniva, C., Hager, A., Amberger, S., Pirker, S.: Models, algorithms and validation for opensource DEM and CFD-DEM. Prog. Comput. Fluid Dyn. 12, 140-152 (2012)

30. Stoddard, S.D.: Identifying clusters in computer experiments on systems of particles. J. Comput. Phys. 27, 291-293 (1978)

31. Kalweit, M., Drikakis, D.: Collision dynamics of nanoscale Lennard-Jones clusters. Phys. Rev. B 74, 235415 (2006)

32. Monaghan, J.J.: Particle methods for hydrodynamics. Comput. Phys. Rep. 3, 71-124 (1985)

33. Monaghan, J.J.: Smoothed particle hydrodynamics. Rep. Prog. Phys. 68, 1703 (2005)

34. Wada, K., Tanaka, H., Suyama, T., Kimura, H., Yamamoto, T.: Collisional growth conditions for dust aggregates. Astrophys. J. 702, 1490 (2009)

35. Planes, M.B., Millan, E.N., Urbassek, H.M., Bringa, E.M.: Stopping of porous projectiles in granular targets. Mon. Notices R. Astron. Soc. Lett. 487, L13-L17 (2019)

36. Katsuragi, H., Durian, D.J.: Unified force law for granular impact cratering. Nat. Phys. 3, 420 (2007)

37. Katsuragi, H.: Physics of Soft Impact and Cratering. Lecture Notes in Physics, vol. 910. Springer, Tokyo (2016)

Publisher's Note Springer Nature remains neutral with regard to jurisdictional claims in published maps and institutional affiliations. 